\title{
ZUM AUSLÄNDERMOTIV IM DEUTSCHEN WITZ. GEZEIGT AM BEISPIEL DER TÜRKENWITZE
}

\section{Zielsetzung}

In diesem Beitrag wird versucht, die Frage nach den Motiven im deutschen Türkenwitz, nach der Rolle der Stereotype bei der Entstehung der Ethnowitze, dem damit zusammenhängenden Problem des guten Geschmacks und nach allen mit den sprachlichen Mitteln des Humoristischen verbundenen Erscheinungen zu beantworten, was zur Folge hat, dass die als Witze über bestimmte Nationen verstandenen Ethnowitze eine konkrete zu nennende Gruppe bilden.

Als Wahlkriterium ist die von den Inhabern der jeweiligen Internetseite ${ }^{2}$ unternommene Einteilung in bestimmte Witzgruppen, unter denen es auch Witze über konkrete Nationen gibt, zu verstehen. Das Analysematerial bilden demzufolge die verschiedenen deutschen Internetseiten entstammenden kurzen 40 Witze über Türken. Alle zitierten Witze stehen in der nicht korrigierten Originalform.

\section{2. „Humor ist, wenn man trotzdem lacht“ (Otto Julius Bierbaum). Zur Funktion des Witzes}

Die nur dem Menschen zugeschriebene Fähigkeit, seine Emotionen mithilfe verschiedener Mittel zum Ausdruck zu bringen, steht bis heute noch im Zentrum des Interesses der Psychologie, wo auf die Zusammenhänge zwischen der Psyche,

\footnotetext{
$1{ }^{*}$ Dr. Katarzyna Sikorska-Bujnowicz, Universität Łódź, Institut für Germanische Philologie, Pomorska 171/173, 90-236 Łódź. E-Mail: k.sikorska_bujnowicz@poczta.onet.pl

${ }^{2}$ Die analysierten Witze entstammen folgenden Internetseiten: http://www.grocceni.com/witze/ witz0859.html, http://www.lachmeister.de/lustige-witze/italiener/index.html, http://www.bfriends. brigitte.de/foren/pavillon/44669-kurze-witze-69.html, http://www.witze.woxikon.de/witze-uni-undschule/320, http://www.witzdestages.net/witze/laender-witze/, http://www.witze-ueber-witze.de/ nationalitaetenwitze.html, http://www.witze-fun.de/witze/witz/4709.
} 
dem Lächeln und dem Lachen hingewiesen wird. Alle Forschungen in diesem Bereich gehen vor allem auf Freud (1905/1985) ${ }^{1}$ und auf Bergson $(1900 / 2011)^{2}$ zurück. Die von dem Menschen als Individuum geknüpften sozialen Kontakte implizieren auch die ihren Ausdruck unter anderem im Lachen wiederfindenden Reaktionen auf alles Erlebte, Gesehene und Gehörte.

Die einem witzigen Text zugeschriebene erzieherische Funktion (Bergson 1900/2011) wird des Weiteren um eine informative ergänzt (Plessner 1941/1982) wobei es in dem letzteren Fall um eine sprachlich nicht zu formulierende Reaktion geht, nämlich um das Lächeln und letztendlich auch das Lachen. Bei Plessner wird dem Ethnowitz der nationale Charakter zugeschrieben, wegen dessen dieser eben als ein Text mit dem Verbreiten des häufig nur mit einer stereotypen Darstellung verbundenen Wissens verstanden wird. Die Vorurteile verschiedener Art sind Ausdruck von der als Tabu zu verstehenden Feindlichkeit den Fremden gegenüber, nicht selten aber auch von der gegen alles Fremde und Unbekannte gerichteten Aggression. Der Witz erfüllt demzufolge seine wichtigste Aufgabe - die der Befriedigung der Aggression und des Triebs dazu, alle momentan geltenden Tabus zu brechen. Damit hängen auch die Fragen nach dem guten Geschmack zusammen, der im Falle der Ausländerwitze nicht selten verletzt wird. Mithilfe der stereotypen Auffassung der jeweiligen Nation, mit Vorurteilen verschiedener Art und nicht selten mit Ekel, Beleidigung und Hass entstehen kurze Witze, bei denen der von Bierbaum ${ }^{4}$ formulierte Spruch „Humor ist, wenn man trotzdem lacht“ zur Geltung kommt.

\section{Zum Stereotyp}

Die Analyse der Ausländerwitze, anders Ethnowitze genannt, setzt die Kenntnis der momentan geltenden Stereotype voraus, die zum richtigen Verstehen, Entschlüsseln und Interpretieren der Witze notwendig ist. Mit dem Verstehen des Humoristischen hängen noch weitere Bereiche zusammen, ohne die es keinen Humor gäbe - Wissen über die Sprache, über die Kultur und das Kontextwissen 5 .

Dem Stereotyp wird eine besondere Funktion verliehen - die des nur eine Interpretation zulassenden Hauptfadens des humoristischen Textes mit einem uni-

${ }^{1}$ S. Freud (1905/1985), Der Witz und seine Beziehung zum Unbewußten, Frankfurt a. M.

2 H. Bergson (1900/2011), Das Lachen. Ein Essay über die Bedeutung des Komischen, Hamburg.

${ }^{3}$ H. Plessner (1941/1982), Lachen und Weinen. Eine Untersuchung nach den Grenzen menschlichen Verhaltens, In: H. Plessner, Gesammelte Schriften VII, Frankfurt a. M., S. 201-387.

${ }^{4}$ Otto Julius Bierbaum (1865-1910) - deutscher Journalist, Schriftsteller, auch als Martin Möbius bekannt.

${ }^{5}$ Mit dem deutschen Ethnowitz setze ich mich u.a. in meiner 2016 erschienenen Monographie aus: vgl. K. Sikorska-Bujnowicz (2016), Zum deutschen Ethnowitz. Eine korpusgestützte Analyse, Łódź. 
versellen Charakter. Die für die Zwecke des Beitrags durchgeführte Analyse stützt sich auf die Auffassung der Ethnowitze als Witze über Nationen.

Als eine Art internationales Gut wird von Frahm (2008, S. 24) ${ }^{6}$ der Ethnowitz aufgefasst, wobei dieser Gruppe der Witze die Zeit- und Raumunabhängigkeit zugeschrieben wird:

Humor lässt sich nicht als sprachliches Phänomen isolieren mit dem Hinweis, er sei mit einem Signifikat namens „nationaler Kulturraum“ identisch - umso weniger, als viele Witze und Anekdoten mühelos Sprach- und Landesgrenzen überwinden und von den Rezipienten nach einer Weile behandelt werden wie ihr eigenes kulturelles Besitztum.

Die überall geltenden und verständlichen Stereotype garantieren die korrekte, von den Autoren der Witze erwartete Interpretation.

Die von Rösch $(2000)^{7}$ erwähnte einerseits individualisierende und andererseits generalisierende Funktion der Stereotype hängt mit der übertriebenen Darstellung bestimmter Nationen und ihrer Mentalität zusammen. Wie in einem Zerrspiegel erscheint dann nicht das im Witz ausgelachte Individuum, sondern die ganze Nation, zu der es gehört.

Diese Art Darstellung hat zwei Erscheinungen zur Folge: einerseits kann ein Witz destruktiv, andererseits aber auch doch konstruktiv wirken. Die mithilfe eines witzigen Textes auszulachenden Nachteile geben dem Rezipienten doch auch die Möglichkeit, alles, was das Fremde und Unbekannte anbetrifft, zusammenzustellen und als Quelle der Informationen über die jeweilige Nation zu betrachten. So kommt es zur Erweiterung des Kulturwissens, die mit der kognitiven Rolle der allgemein geltenden Stereotype zusammenhängt.

Die auch dank den Witzen verbreiteten Informationen über Fremde beeinflussen die Wahrnehmung einer anderen Nation und die Einstellung zu ihr. Dabei wird auch eine im Rahmen einer multikulturellen Gesellschaft lebende ethnische Minderheit als fremd empfunden und diese wird auch unter dem Begriff Nation als Zielscheibe der Witze aufgefasst. Der multikulturelle Charakter einer Gesellschaft ermöglicht den unmittelbaren Kontakt mit dem mit einer anderen Nation verbundenen Fremden, was doch auch die Rolle des Neuen übernimmt, sodass auf diese Weise einerseits die Zusammengehörigkeitsgefühle gestärkt werden und andererseits die Anerkennung einer anderen Nation zustande kommt.

Mit dem als Mittel des Ausländerwitzes gedachten Stereotyp kommt es auch zur Integration. Diese ist dem Lernprozess und dem Akzeptieren zu verdanken, die als Resultate der sich zwischen verschiedenen Nationen entwickelnden Rela-

${ }^{6}$ T. Frahm (2008), Verneigung vor dem Säbel. Gibt es einen bulgarischen Humor?, In: T. Hoffmann, M. Ch., A. Lercher Middeke, K. Tittel (Hrsg.), Humor, Göttingen, S. 21-34.

7 O. Rösch (Hrsg.) (2000), Stereotypisierung des Fremden. Auswirkungen in der Kommunikation, Berlin, S. 85-112. 
tionen gesehen werden sollen. Auf diese Weise spielen die Stereotype auch eine soziale Rolle. Die Witze über Ausländer und ihren Alltag können sogar zur Verständigung von verschiedenen Nationen beitragen.

\section{Zum Ausländerwitz ${ }^{8}$}

Ein als ein humoristischer aufzufassender Text soll zweierlei Kriterien entsprechen - der Semantik und der Struktur. Die genannten Punkte beziehen sich auf die von Attardo und Raskin im Jahre 1991 formulierte General Theory of Verbal Humour ${ }^{9}$, die die Rolle der das Umschalten der Skripts ermöglichenden logischen Regeln und die des unter der Witzszene verstandenen situativen Kontextes betont. Die Struktur des Witzes, vor allem seine Zielscheibe, die Witzgattung und die Form spielen eine genauso wichtige Rolle wie die Semantik des angebotenen Textes. Es wird dabei davon ausgegangen, dass der Rezipient über das notwendige Kulturwissen verfügt, das ihm das richtige Verstehen und Interpretieren der Witze ermöglicht.

Die den Rezipienten angebotenen Ausländerwitze sprechen alle für die jeweilige Nation wichtigen Themen an, unter denen an erster Stelle das Zugehörigkeitsgefühl genannt werden soll, das neben den Kultur- und Religionsfragen erscheint. Alles soll so eindeutig sein, dass es dem Leser bzw. Zuhörer leichtfällt, auch die mehrdeutigen sprachlichen Kontexte richtig zu verstehen.

Der Einfluss der Sprache einer sozialen Gruppe auf den Witz wird von Raskin $(1985)^{10}$ betont, was zur Folge hat, dass auch die Witze voll von Elementen der Gruppensprachen sind.

Die informative Funktion der Witze geht auf die für die jeweilige Nation charakteristischen Eigenschaften zurück. Sie bewirkt den bewussten Einsatz und andererseits auch den vom Tabu abhängigen Verbot aller Inhalte, die in einem Witz als Thema, Zielscheibe bzw. Motiv auftreten könnten. Wird trotzdem das Tabu gebrochen, ist in diesem Fall die Rede von schlechtem Geschmack, wobei doch der Text an sich weiter als Witz lebt, gleich nach dem schon von Bierbaum formulierten Spruch „Humor ist, wenn man trotzdem lacht“.

Das das Äußerungsbild der Nationen skizzierende Nonverbale ist auch mit der Sprache verbunden, sodass zwischen den Zeilen gelesen werden kann. Die

\footnotetext{
${ }^{8}$ Ausländerwitze werden auch Ethnowitze genannt. Ich beschränke mich jedoch auf die Witze über eine Nation, wobei als Ethnowitze auch Witze von konkreten Nationen definiert werden. Vgl. dazu mehr: A. Middeke, S. Murdsheva (2008) Nationen- und Ethnowitze im interkulturellen DaFUnterricht, In: T. Hoffmann, M. Ch. Lercher, A. Middeke, K. Tittel (Hrsg.), Humor, Göttingen, S. 221-232

${ }^{9}$ S. Attardo, V. Raskin (1991), Script theory revis(it)ed: Joke similarity and joke representation model, In: Humor - International Journal of Humor Research, Volume 4, Issue 3-4, S. 293-349.

${ }^{10}$ V. Raskin (1985), Semantic Mechanism of Humour, Dordrecht.
} 
fehlende Sprachkenntnis kann dabei zu den nicht selten komischen Missverständnissen verschiedener Art führen. Von Driessen (1999) ${ }^{11}$ wird darauf hingewiesen, dass die Kenntnis der Sprache der Witzautoren den Einblick in die jeweilige Kulturwelt ermöglicht, was zusätzlich bedeutet, dass der Rezipient imstande ist, alle versteckten Inhalte zu finden und zu dekodieren. So wird das Fremde zum Bekannten. Von dem Witzrezipienten wird verlangt, dass er die Sprache der Witzautoren so gut versteht, dass er imstande ist, selbst das Wortspiel zu spielen, indem er u.a. polyseme Ausdrücke, übertragene Bedeutung oder aber auch die für die Zwecke des Humoristischen eingesetzten fehlerhaften Formen nicht nur wortwörtlich versteht, sondern auch ihre Funktion im jeweiligen Witz erkennt.

Die Ausländerwitze von heute bedeuten nicht selten einen mit einem negativen Bild der jeweiligen Nation verbundenen Witz. Diese Erscheinung kann mit der Überlegenheitstheorie (Bergson 1900/2011) erklärt werden, in der der Humor zur Bestrafung und zur Erniedrigung asozialer Menschen dient. Auf diese Weise können Witze als Mittel zum Ausdruck der negativen Gefühle verstanden werden und zugleich wird ihnen die im Mittelpunkt der Entlastungstheorie stehende Ventilfunktion zugeschrieben ${ }^{12}$.

Im Falle der Ausländerwitze liegt eine Mischung aus Sachwitz und Sprachwitz vor. Die übertriebene Darstellung der mit dem Kampf gegen Tabu und soziale Normen verbundenen Situationen charakterisiert diese Witze als Sachwitze. Das dem situativen Kontext angepasste Sprachliche ergänzt alles Nonverbale, sodass im überraschenden Ende die Pointe zu finden ist. Erst die Kenntnis beider Kontexte gibt dem Rezipienten die Möglichkeit, den Witz als eine Einheit zu verstehen. Dabei muss auch darauf hingewiesen werden, dass die eventuell vorhandenen fehlenden Sprachkenntnisse kein Störfaktor sind, was auf die Kenntnisse von allem Nonverbalen zurückgeht. Zum korrekten Verstehen der Witze über Nationen werden politische, soziale, kulturelle und religiöse Kenntnisse verlangt, damit sie trotz der fehlenden Sprachkenntnisse ihre Witzfunktion ausüben. Dennoch muss betont werden, dass das richtige Witzverstehen sowohl mit dem situativen als auch mit dem sprachlichen Kontext zusammenhängt. Die Interpretation der humoristischen Texte ist jedoch vom Rezipienten abhängig. Die nicht selten im Witz ausgenutzte Polysemie kann zur Bildung von mehreren Kontexten führen, sodass nicht nur eine, sondern viele Interpretationen zugelassen sind.

${ }^{11}$ H. Driessen (1999), Lachen und Feldforschung: Betrachtungen aus dem Blickwinkel der Ethnologie, In: J. Bremmer, H. Roodenburg (Hrsg.), Kulturgeschichte des Humors, Darmstadt, S. $167-185$.

${ }^{12}$ Vgl. mehr zu den Humortheorien: K. Sikorska-Bujnowicz (2013), Sprachliche Mittel des Humoristischen im Deutschen und im Polnischen, Łódź, S. 23-28. 


\section{Zum deutschen Türkenwitz. Ergebnisse der Analyse}

Aus einer Unmenge der Internetwitze wurden für die Zwecke der Analyse 40 Türkenwitze gewählt. Die genannte Gruppe wurde unter dem Aspekt der Form und der Sprache analysiert. Es war von Bedeutung, welche sprachlichen Mittel des Humoristischen eingesetzt wurden. Die Autoren der Witze bedienen sich oft der absichtlich benutzten fehlerhaften Formen verschiedener Art. Es sind u.a. Fehler auf der morphologischen Ebene und die im Bereich der Syntax.

Bei der Besprechung der Internetwitze soll vor allem auf ihre Anonymität hingewiesen werden, was zur Folge hat, dass die Autoren nicht selten die Grenzen verschiedener Art überschreiten, sodass es zu den Verstößen gegen soziale Normen kommt. Es wird relativ oft mit der Beleidigung gespielt (Witz 2, 11, 17, 18, 23, 24, 26, 27, 28, 29, 30, 31, 33, 40), wenn man z.B. die Türken mit dem Müll vergleicht, den man loswerden will (Witz 28, 29, 30, 31). Das Motiv des Willens, Türken aus Deutschland wegzujagen, steht oft in den makabren Witzen (Witz 36, 37, 38, 39). Im Witz 40 wird sogar eindeutig gezeigt, welche Einstellung die Deutschen zu ihren türkischen Nachbarn haben.

In den Türkenwitzen erscheinen nicht selten mehrere Vorurteile. Das Motiv eines faulen, asozialen und auf Kosten des Staates lebenden Türken steht neben dem der für ihn charakteristischen fehlenden Sprachkenntnisse.

In 15 von 40 für die Zwecke dieses Beitrags analysierten Türkenwitzen fällt auch schwarzer Humor auf (Witz 2, 8, 9, 10, 11, 12, 13, 17, 22, 28, 35, 36, 37, 38, 39).

\subsection{Deutscher Türkenwitz. Form, Motive und Sprache}

$\mathrm{Zu}$ den beliebten Motiven der Ausländerwitze allgemein gehört die Faulheit. Die Analyse hat gezeigt, dass es selten der Fall ist, dass in den Witzen nur ein Motiv auftaucht, denn es erscheinen oft mindestens zwei, was an folgenden Beispielen gut erkennbar ist:

(1) Warum gibt es so viele Türken in Deutschland?

Weil die in der Türkei gehört haben, dass es in Deutschland nicht so viel Arbeit geben soll.

1.1. Frage-Antwort-Form

1.2. Warum-Frage

(2) Wie lässt du einen Türken hungern?

Versteck den Sozialhilfe-Scheck unter seine Arbeitsschuhe!

2.1. Frage-Antwort-Form

2.2. Wie-Frage

2.3. schwarzer Humor

2.4. Beleidigung 
(3) Kommt ein Türke aufs Arbeitsamt. Sagt: „Ich will Arbeit.“ Sagt der Mann hinter dem Schalter: „Du kannst eine Villa haben, einen Ferrari und eine geile Blondine noch dazu“.

Der Türke: „Willst du mich verarschen?“ „Wer hat denn angefangen mit verarschen?!?"“

\subsection{Dialog-Form}

3.2. Spiel mit dem situativen Kontext

(4) Warum taucht auf der Enterprise nie ein Türke auf?

Die arbeiten in der Zukunft auch nicht.

4.1. Frage-Antwort-Form

4.2. Warum-Frage

Es gibt Witze mit dem Motiv der Faulheit und der Veranlagung zur Kriminalität

(5) Eine türkische Frau erwartet Zwillinge. Als es soweit ist und die Wehen einsetzen, ruft sie im Krankenhaus an. Die Zentrale erklärt ihr aber, dass sie zur Zeit keinen freien Krankenwagen hätten, ihnen aber die Polizei vorbei schicken würden, die in solchen Fällen geübt wäre. Gesagt, getan. Wenige Stunden später, kurz vor der Geburt, ruft der angekommene Polizist: „Pressen, pressen ..." Auf einmal sieht man einen kleinen Kopf hervorschauen, der aber schnell wieder zurückweicht und sagt: „Ey, Murat, wir müssen hinten raus, vorne stehen die Bullen!“

5.1. Erzählung + Dialog-Form

5.2. Stereotyp eines kriminellen Türken

5.3. Veranlagung zur Kriminalität

5.4. Sprache

5.4.1. Element des Türkisch-Deutschen Ey

5.4.2. Gebrauch einer umgangssprachlichen abwertenden Bezeichnung für einen Polizisten Bulle

(6) Was macht ein Türke beim Arbeitsamt? Einbrechen!

6.1. Frage-Antwort-Form

6.2. Was-Frage

(7) Was sagt man zu einem Türken im Anzug?

„Der Angeklagte möge sich bitte erheben.“

7.1. Frage-Antwort-Form

7.2. Was-Frage

(8) Was ist der Unterschied zwischen guten und schlechten Türken?

Gute Türken sind in mittelbewachten Gefängnissen. 


\subsection{Frage-Antwort-Form}

8.2. Was-Frage

8.3. schwarzer Humor

(9) Warum solltest du einen Türken, wenn er vor dir auf einem Fahrrad fährt, nicht überfahren?

Es könnte dein Fahrrad sein!

9.1. Frage-Antwort-Form

9.2. Warum-Frage

9.3. schwarzer Humor

(10) Wo geht es hin, wenn ein Deutscher einen Türken fährt? Ins Gefängnis!

10.1. Frage-Antwort-Form

10.2. Wohin-Frage

10.3. schwarzer Humor

(11) Was passiert, wenn eine Türkin abtreibt?

Die Kriminalpolizei schickt ihr einen Scheck.

11.1. Frage-Antwort-Form

11.2. Was-Frage

11.3. derber, vulgärer Witz

11.4. schwarzer Humor

11.5. Beleidigung

In den deutschen Witzen über Türken erscheint auch die Gewalttätigkeit als Motiv, z.B.

(12) Kommt ein Türke in einen Waffenladen. Fragt er: (10.1.) „Haben Messer?“ „Nee.“ „Haben Pistolen?“ „Nee.“ „Haben Handgranate?“ „Nee.“ Geht der Türke wieder raus, und sieht alle möglichen Waffen im Schaufenster. Da überlegt er, geht wieder rein und fragt: „Haben was gegen Türken?““ „Ja, Messer, Pistolen, Handgranaten..."

12.1. Dialog-Form

12.2. Spiel mit dem situativen Kontext

12.3. schwarzer Humor - Gewalttätigkeit der Türken

12.4. Anspielung auf die Ausländerfeindlichkeit der Deutschen den Türken gegenüber

12.5. Sprache

12.5.1. fehlende Personalpronomina als Subjekte *Haben Messer/Pistolen/Handgranate?, *Haben was gegen Türken? 
(13) Wer sind die freundlichsten Menschen?

Die Türken. Sie kommen zu zehnt und fragen: „Has du Problem?“

13.1. Frage-Antwort-Form

13.2. Wer-Frage

13.3. schwarzer Humor

13.4. Sprache

13.4.1. fehlerhafte Formen:

13.4.1. falsche Konjugationsform *has du

13.4.2. fehlender Artikel *Has du Problem?

Neben den genannten Motiven erscheint in den Türkenwitzen ein intertextuelles Element, nämlich der Name der deutschen Discountergeschäftskette Aldi. Das Geschäft wird von dem Türken für ein Wunderland gehalten und die dort beim Einkauf verbrachten Zeiten als gute Zeiten betrachtet:

(14) Was heißt auf Türkisch „Alice im Wunderland“?

Ibrahim im Aldi.

14.1. Frage-Antwort-Form

14.2. Was-Frage

14.3. Sprache

14.3.1. intertextuelles Element - Buchtitel „Alice im Wunderland“"

14.3.2. intertextuelles Element - Name der deutschen Discounterkette Aldi

(15) Warum feiern Türken kein Erntedankfest?

Aldi hat am Sonntag geschlossen.

15.1. Frage-Antwort-Form

15.2. Warum-Frage

15.4. Sprache

15.4.1. intertextuelles Element - Name der deutschen Discounterkette Aldi

(16) Wie heißen „Gute Zeiten, schlechte Zeiten“ auf Türkisch?

Aldi auf, Aldi zu!

16.1. Frage-Antwort-Form

16.2. Wie-Frage

16.3. Sprache

16.3.1. intertextuelles Element - Titel der deutschen Fernsehserie „, Gute Zeiten schlechte Zeiten“

16.3.2. intertextuelles Element - Name einer deutschen Discounterkette $A l d i$ 
Das Motiv der Arbeit erscheint zwar in den Türkenwitzen, es wird aber in Verbindung mit dem Zweifel an den Fähigkeiten der Türken gesetzt, sodass es u.a. in solchen Witzen zu sehen ist:

(17) Was kommt raus, wenn man eine Türkin mit einer Spinne kreuzt?

Eine achtarmige Putzfrau!

17.1. Frage-Antwort-Form

17.2. Was-Frage

17.3. Beleidigung

17.4. schwarzer Humor

(18) Was macht ein Türke in der Uni? Er putzt!

18.1. Frage-Antwort-Form

18.2. Was-Frage

18.3. Beleidigung

(19) Was sagt ein Türke ohne Arbeit zu einem Türken mit Arbeit?

Einmal Currywurst mit Pommes!

19.1. Frage-Antwort-Form

19.2. Was-Frage

In Verbindung mit dem in den Witzen auftauchenden Stereotyp eines türkischen Machos erscheinen sexuelle Motive:

(20) Was heißt Gruppensex auf Türkisch?

Kümmelgetümmel!

20.1. Frage-Antwort-Form

20.2. Was-Frage

20.3. Sprache

20.3.1. Determinativkompositum Kümmelgetümmel als Spiel mit der Wortbildung in Anlehnung an das von Türken in der Küche gebrauchte Gewürz

20.3.2. Bezeichnungsübertragung

(21) Woran erkennt man eine türkische Domina?

Am Lederkopftuch!

21.1. Frage-Antwort-Form

21.2. Woran-Frage

21.3. Sprache

21.3.1. Determinativkompositum Lederkopftuch als Wortbildungsspiel in Anlehnung an die mit einer Domina zusammenhängenden Assoziationen 
Nicht selten werden die Türken als große Angeber ausgelacht, z.B.

(22) Was ist ein Türke mit einem Messer im Rücken?

Guter Türke.

Und mit zwei Messern?

Angeber!

22.1. Frage-Antwort-Form

22.2. Stereotyp eines gewalttätigen Türken

22.3. schwarzer Humor

Die Türken werden zur Zielscheibe, weil ihnen vorgeworfen wird, dass sie gar nicht an ihre Hygiene denken, z.B.

(23) Wie nennt man 5 Türken in einem VW?

Stinkkäfer.

23.1. Frage-Antwort-Form

23.2. Wie-Frage

23.3. Beleidigung

23.4. geschmackloser Witz

23.5. Sprache

23.5.1. Wortspiel mit Stinkkäfer - VW-Käfer vs. Stinkkäfer

(24) Warum tragen Türken Kapuzen-Pullover?

Um die Floh-Halsbänder zu verbergen!

24.1. Frage-Antwort-Form

24.2. Warum-Frage

24.2. Beleidigung

24.3. geschmackloser Witz

(25) Die türkische Nationalmannschaft hat ein Länderspiel gewonnen.

Der Trainer in der Kabine: „So Jungs, jetzt haben wir uns eine

Erfrischung verdient. Ercan, mach das Fenster auf!“”

25.1. Erzählung + Monolog-Form

25.2. Beleidigung

25.3. geschmackloser Witz

(26) Was passiert, wenn ein Türke seinen Kopf im Meer hält?

Ölpest!

26.1. Frage-Antwort-Form

26.2. Was-Frage 
26.3. Beleidigung

26.4. geschmackloser Witz

(27) Wieso fliegen Tauben über eine Türkenwohnung immer im Kreis?

Weil sie mit einem Flügel die Nase zuhalten müssen!

27.1. Frage-Antwort-Form

27.2. Warum/Wieso-Frage

27.3. Beleidigung

27.4. geschmackloser Witz

(28) Was ist eine Mülltonne auf Rädern? Türkischer Karnevalswagen...

28.1. Frage-Antwort-Form

28.2. Was-Frage

28.3. Beleidigung

28.4. schwarzer Humor

28.5. geschmackloser Witz

(29) Was ist eine Mülltonne vor einer Ampel? Türkendisco

29.1. Frage-Antwort-Form

29.2. Was-Frage

29.3. Beleidigung

29.4. Sprache

29.4.1. Determinativkompositum Türkendisco

(30) Was ist eine Mülltonne in der Gosse?

Ein Türkenwohnheim.

30.1. Frage-Antwort-Form

30.2. Was-Frage

30.3. Beleidigung

30.4. geschmackloser Witz

30.5. Sprache

30.5.1. Determinativkompositum Türkenwohnheim

(31) Warum sind am Strand einige Mülltonnen mit einem roten Halbmond gekennzeichnet?

Damit die Türken ihre Strandkörbe erkennen.

31.1. Frage-Antwort-Form

31.2. Warum-Frage

31.3. Beleidigung

31.4. geschmackloser Witz 
Das Kopftuch als Zeichen der kulturellen und nationalen Zugehörigkeit erscheint als Motiv in folgenden Witzen:

(32) Was ist grün und trägt ein Kopftuch?

'ne Gürkin

32.1. Frage-Antwort-Form

32.2. Was-Frage

32.3. Sprache

32.3.1. Kontaminationsform aus Gurke und Türkin

(33) Warum tragen Türkinnen Kopftücher?

Damit man sie von ihren Männern unterscheiden kann.

33.1. Frage-Antwort-Form

33.2. Warum-Frage

33.3. derber Witz

33.4. Beleidigung - die in Frage gestellte Schönheit der Türkinnen

33.5. geschmackloser Witz

Dieses Wiedererkennungszeichen wird auch auf homosexuelle Männer übertragen:

(34) Woran erkennt man einen schwulen Türken?

Am Kopftuch!

34.1. Frage-Antwort-Form

34.2. Woran-Frage

34.3. geschmackloser Witz

Nicht selten kommt es vor, dass die Türken als diese Nation dargestellt werden, die jede Gelegenheit dazu nutzt, Geld zu verdienen, z.B.:

(35) Ein Türke in einem Beerdigungsinstitut: „Gut Tag! Brauchen Grabstein. Drauf schreiben: Ali tot.“ „Guter Mann, bei uns ist es üblich, dass man ein paar Worte mehr auf den Grabstein schreibt!“ „Is gut: Ali tot, verkaufen Ford Transit!“

35.1. Dialog-Form

35.2. schwarzer Humor

35.3. fehlerhafte Formen:

35.3.1. falsche Begrüßungsformel * Gut Tag!

35.3.2. Infinitivgruppe statt eines Satzes * drauf schreiben

35.3.3. fehlender Prädikatsteil * Ali tot

35.3.4. falsche Verbformen *is, *verkaufen 
Zum Schluss sollen noch Witze zitiert werden, in denen die deutsche Ausländerfeindlichkeit den Türken gegenüber zum Ausdruck gebracht wird, z.B.:

(36) Wie lange darf man Türken mit einem Auge ansehen?

Bis das Magazin leer ist.

36.1. Frage-Antwort-Form

36.2. Wie lange-Frage

36.3. schwarzer Humor, makabrer Witz

(37) In Berlin hat man gerade ein Geschäft geschlossen, auf dem stand:

„Wir bedienen lieber 1000 Türken als einen Deutschen“. Es war ein

Bestattungsunternehmen.

37.1. Erzählung-Form

37.2. schwarzer Humor, makabrer Witz

(38) Ein Mann kommt mit defekten Stoßdämpfern in eine Werkstatt. Der Meister wechselt ihm die Stoßdämpfer aus, und alles ist wunderbar ....Prompt 2 Wochen später kommt er wieder, und es sind schon wieder die Stoßdämpfer, die kaputt sind. Da nimmt der Meister ihn beiseite: „Sagen Sie mal, nicht das ich mich beschwere, ich verdiene ja schließlich dran, aber wie haben Sie das eigentlich hin bekommen, dass die schon wieder kaputt sind?““ „Naja, ich habe einen Türken überfahren.“ „Na, hören Sie mal, von so einem Türken wird doch wohl der Stoßdämpfer nicht kaputt gehen!“” „Doch schon, er wollte übers Feld flüchten!“‘

38.1. Erzählung-Form

38.2. schwarzer Humor

38.3. Spiel mit dem situativen Kontext

(39) Wird ein toter Türke in einem Waldstück mit 23 Schusswunden aufgefunden. Sagt der eine Polizist: „Mensch, hast Du schon mal so einen brutalen Selbstmord gesehen?"

39.1. Erzählung + Dialog-Form

39.2. schwarzer Humor, makabrer Witz oder eindeutig auf die Einstellung der Deutschen zu den Türken gezeigt wird:

(40) Vor welchen Worten haben Deutsche am meisten Angst?

„Hey, Mann, wir sind deine neuen Nachbarn!“

40.1. Frage-Antwort-Form

40.2. derber Witz

40.3. Beleidigung 


\subsection{Ergebnisse der Analyse}

Die Analyse hat gezeigt, dass die meisten von den besprochenen Witzen die Frage-Antwort-Form haben (32/40), des Weiteren sind folgende Formen zu nennen: Dialog (3/40), Erzählung+Dialog (3/40), Erzählung (1/40) und Erzählung+Monolog (1/40).

Interessant scheint die Tatsache zu sein, welche Form dann die Fragen in den genannten Frage-Antwort-Witzen haben, d.h. die meisten Fragesätze sind dann die Was-Fragen (15/32) und Warum-Fragen (8/32), weiter erscheinen die Wie-Fragen (3/32), Woran-Fragen (2/32), wobei am seltensten die Wohin-Fragen (1/32), Wer-Fragen (1/32), Wie lange-Fragen (1/32) und Wovor-Fragen $(1 / 32)$ zu finden sind.

Die Analyse der Sprache der Witze hat ergeben, dass am meisten mit der Wortbildung gespielt wird (Witz 20: Kümmelgetümmel, Witz 21: Lederkopftuch, Witz 29: Türkendisco, Witz 30: Türkenwohnheim), weil das deutsche Wortbildungsmodell des Determinativkompositums aus sprachökonomischen Gründen am produktivsten ist und im Falle der kurzen humoristischen Texte spielt es eine äußerst wichtige Rolle, viel Inhalt mit relativ wenig Lexik zum Ausdruck zu bringen. In einem Text (Witz 32) kommt auch die Kontamination zum Vorschein, indem aus Gurke und Türkin das Wort Gürkin gebildet wird. Die Witzautoren greifen zum polysemen Wortschatz, dessen Kenntnis für das richtige Verstehen des angebotenen Textes von Bedeutung ist (wie im Witz 23: $V W$-Käfer vs. Stinkkäfer). Man bedient sich auch der Bezeichnungsübertragung (Witz 20: Kümmelgetümmel).

Das erwartete Interpretieren der Witze setzt die Kenntnis der Sprachvarietäten voraus, sei es die der Umgangssprache (Witz 5: Bulle), sei es die des Türkisch-Deutschen (Witz 5: Anredeform Ey).

Nicht weniger wichtig sind dabei alle in den Witzen erscheinenden intertextuellen Elemente, die den über ihre Kenntnis verfügenden Rezipienten alles korrekt verstehen und interpretieren lassen (Witz 14: „Alice im Wunderland“, Aldi; Witz 15: Aldi; Witz 16: „, Gute Zeiten, schlechte Zeiten “, Aldi).

Für die Zwecke des Humoristischen werden auch fehlerhafte Formen gebraucht, die die morphologische und syntaktische Ebene betreffen (Witz 12: fehlende Personalpronomina als Subjekte *Haben Messer/Pistolen/Handgranate?, *Haben was gegen Türken?; Witz 13: falsche Konjugationsform *has du, fehlender Artikel *Has du Problem?; Witz 35: falsche Begrüßungsformel *Gut Tag!, Infinitivgruppe statt eines Satzes *drauf schreiben, fehlender Prädikatsteil *Ali tot, falsche Verbformen *is, *verkaufen). 


\section{Fazit}

Die Witze über Türken werden relativ oft mit der Faulheit und dem Leben auf Kosten des die Einwanderer empfangenden Staates assoziiert. Die Stereotype verschiedener Art werden zusätzlich mit dem Einsatz fehlerhafter Formen unterstützt, sodass sich im Endeffekt ein eher trauriges Bild eines Einwanderers, unabhängig von der Nation, ergibt. Man darf nicht vergessen, dass eben die Anonymität die Quelle einer solchen Einstellung ist, dass mit den nicht selten mit Hass und Ausländerfeindlichkeit gefüllten Ausländerwitzen die Grenzen des guten Geschmacks sehr leicht und schnell überschritten werden.

\section{LITERATURVERZEICHNIS}

Attardo S., Raskin V. (1991), Script Theory Revis(it)ed: Joke Similarity ans Joke Representation Model. In: Humor 3: 3/4, S. 293-347.

Bergson H. (1900/1977), Śmiech. Esej o komizmie, polnische Übersetzung von S. Cichowicz, Kraków.

Bergson H. (1900/2011), Das Lachen. Ein Essay über die Bedeutung des Komischen, Hamburg.

Dimova A. (2008), Humor und Witz als Übersetzungsproblem. In: T. Hoffmann, M.-Ch. Lercher, A. Middeke, K. Tittel (Hrsg.): Humor - Grenzüberschreitende Spielarten eines kulturellen Phänomens, Göttingen, S. 7-20.

Driessen H. (1999), Lachen und Feldforschung: Betrachtungen aus dem Blickwinkel der Ethnologie. In: J. Bremmer, H. Roodenburg (Hrsg.), Kulturgeschichte des Humors, Darmstadt, S. $167-185$.

Frahm T. (2008), Verneigung vor dem Säbel. Gibt es einen bulgarischen Humor? In: Hoffmann T., Lercher M.Ch., Middeke A., Tittel K. (Hrsg.), Humor - Grenzüberschreitende Spielarten eines kulturellen Phänomens, Göttingen, S. 21-34.

Freud S. (1905/1993), Dowcip i jego stosunek do nieświadomości, Warszawa.

Freud S. (1905/1985), Der Witz und seine Beziehung zum Unbewußten, Frankfurt a. M., A. Middeke, S. Murdsheva (2008), Nationen- und Ethnowitze im interkulturellen DaFUnterricht. In: T. Hoffmann, M. Ch. Lercher, A. Middeke, K. Tittel (Hrsg.), Humor - Grenzüberschreitende Spielarten eines kulturellen Phänomens, Göttingen, S. 221-232

Plessner H. (1941/1982), Lachen und Weinen. Eine Untersuchung nach den Grenzen menschlichen Verhaltens. In: H. Plessner, Gesammelte Schriften VII, Frankfurt a. M., S. 201-387.

Raskin V. (1985), Semantic Mechanism of Humour, Dordrecht.

Rösch O. (Hrsg.) (2000), Stereotypisierung des Fremden. Auswirkungen in der Kommunikation, Berlin, S. 85-112.

Sikorska-Bujnowicz K. (2013), Sprachliche Mittel des Humoristischen im Deutschen und im Polnischen, Łódź.

Sikorska-Bujnowicz K. (2016), Zum deutschen Ethnowitz. Eine korpusgestützte Analyse, Łódź. 


\title{
INTERNETQUELLEN
}

http://www.grocceni.com/witze/witz0859.html.

http://www.lachmeister.de/lustige-witze/italiener/index.html.

http://www.bfriends.brigitte.de/foren/pavillon/44669-kurze-witze-69.html.

http://www.witze.woxikon.de/witze-uni-und-schule/320.

http://www.witzdestages.net/witze/laender-witze/.

http://www.witze-ueber-witze.de/nationalitaetenwitze.html.

http://www.witze-fun.de/witze/witz/4709.

Katarzyna Sikorska-Bujnowicz

\section{FOREIGNER THEMES IN THE GERMAN JOKE. BASED ON THE EXAMPLE OF JOKES ABOUT TURKS}

\begin{abstract}
(Summary)
The article attempts to answer the question of what the themes of German jokes about Turks are and what role stereotypes play. The form of the joke and all means used to build humour, primarily incorrect forms used intentionally, are also important.
\end{abstract}

Keywords: humour research, German ethnic joke, stereotype in the joke. 